\title{
Fundo Estratégico da Organização Pan-Americana da Saúde: mecanismo facilitador para melhorar 0 acesso aos medicamentos ${ }^{1}$
}

\author{
Myrza M. L. de L. Horst ${ }^{2}$ e Orenzio Soler ${ }^{3}$
}

Como citar Horst MMLL, Soler O. Fundo estratégico da Organização Pan-Americana da Saúde: mecanismo facilitador para melhorar o acesso aos medicamentos. Rev Panam Salud Publica. 2010;27(1):43-8.

RESUMO Objetivo. Descrever o funcionamento do Fundo Estratégico para medicamentos da Organização Pan-Americana da Saúde (OPAS).

Método. Estudo retrospectivo, com recorte temporal de 2004 a 2007, fundamentado em levantamento bibliográfico e documental em arquivos dos escritórios da OPAS nos Estados Unidos, El Salvador, Guatemala, Honduras e Brasil. Foram levantados o volume total de recursos e os tipos de medicamentos movimentados por meio do Fundo Estratégico nos anos de 2004, 2005, 2006 e nos meses de janeiro a setembro de 2007.

Resultados. O levantamento revelou um fundo bem estruturado que movimentou recursos crescentes, de US\$ 3475 043,00 em 2004 a US\$ 19646 634,00 em 2007 (de janeiro a setembro). Houve um crescimento na participação dos antirretrovirais nesse dispêndio, de pouco menos de $8 \%$ do total em 2004 a 57,89\% em 2007. Ainda em 2007, 66,63\% da movimentação financeira do Fundo Estratégico foram destinados à compra de antirretrovirais para nove países (Brasil, Guatemala, El Salvador, Equador, Honduras, Haiti, Belize, Nicarágua e Bolívia) de um total de 17 países participantes. O país que mais movimentou recursos financeiros por meio do Fundo Estratégico foi o Brasil, com $63 \%$ dos gastos para compra de insumos estratégicos no período de janeiro a setembro de 2007.

Conclusão. A proposta de unificar as compras de medicamentos de todos os países participantes para melhor negociação de preços e administração das compras permitirá economias de escala. O Fundo Estratégico pode contribuir para ampliar o acesso aos medicamentos e melhorar a gestão do sistema público de saúde da América Latina.

Palavras-chave Medicamentos essenciais; insumos farmacêuticos; cooperação técnica; equidade no acesso; América Latina.

1 Este estudo é parte da dissertação apresentada em 2007 por Myrza M. L. de L. Horst à Organización Iberoamericana de Seguridad Social e à Universidad de Alcalá, Espanha, para obtenção do grau de mestre.

2 Organização Pan-Americana da Saúde, Unidade de Medicamentos, Tecnologia e Pesquisa, Brasília (DF), Brasil. E-mail: myrza@bra.ops-oms.org

3 Universidade Federal do Rio de Janeiro, Faculdade de Farmácia, Departamento de Medicamentos, Programa de Farmácia Social. Correspondência: Centro de Ciências da Saúde (CCS), Bloco K, Sala 050, Ilha do Fundão, CEP 21940-590, Cidade Universitária, Rio de Janeiro, RJ, Brasil. Tel.: +5521-2562.6444; e-mail: orenzio@pharma.ufrj.br, orenziosoler@gmail.com
Na América Latina, a metade de uma população de cerca de 560 milhões de habitantes não está coberta por mecanismos de proteção social. Além disso, mais de 100 milhões de pessoas não têm acesso aos serviços de saúde, nem mesmo à água potável ou ao saneamento básico, por razões econômicas ou geográficas. As tentativas dos sistemas e serviços de saúde de assegurar o acesso aos insumos essenciais se confrontam com um padrão de iniquidade que traz à re- gião o desequilíbrio, a extrema concentração de renda e injustiças evidentes (1), e que se estende ao acesso aos serviços de saúde (2).

$\mathrm{O}$ acesso a medicamentos essenciais tem sido tradicionalmente considerado uma parte fundamental de toda a política farmacêutica. Como tal, torna-se um elemento substantivo na elaboração e execução de ações ou programas sanitários cujo objetivo é assegurar o grau máximo de saúde para todo ser humano, re- 
conhecido como fundamental na constituição da Organização Mundial da Saúde (OMS) e reafirmado em diversas declarações, convenções e tratados regionais ou internacionais $(3,4)$.

No ano de 2000, a pedido dos EstadosMembros, a Organização Pan-Americana da Saúde (OPAS) criou o Fundo Rotatório Regional para Provisões Estratégicas de Saúde Pública (4), conhecido como Fundo Estratégico. Essa iniciativa teve o objetivo de facilitar as compras de medicamentos e insumos estratégicos, apoiando os países na melhoria do acesso a medicamentos essenciais usados na infecção pelo vírus da imunodeficiência humana (HIV), tuberculose, malária e leishmaniose (5). São 17 os países participantes do Fundo Estratégico: Barbados, Belize, Bolívia, Brasil, Equador, El Salvador, Guatemala, Haiti, Honduras, Jamaica, Nicarágua, Panamá, Paraguai, Peru, República Dominicana, Suriname e Trinidad e Tobago. Desde sua criação, o Fundo tem facilitado a compra a baixo custo de provisões prioritárias para a saúde pública, assim como, paralelamente, capacitado pessoas para a gestão da logística do medicamento $(5,6)$.

Inicialmente, o Fundo Estratégico baseou-se na experiência exitosa do Programa Ampliado de Imunização, que presta apoio aos Estados-Membros na seleção, compra, distribuição e uso de vacinas por meio do Fundo Rotatório para Compra de Vacinas da OPAS/OMS (4). Além disso, o Fundo Estratégico buscou qualitativamente um enfoque voltado para facilidades administrativas e processo de compras de medicamentos e insumos estratégicos de fornecedores pré-qualificados pela OPAS/OMS. Utiliza-se a estratégia de consolidar todas as demandas de cada país participante, a fim de que as compras sejam efetuadas em grande quantidade e com menor preço, garantindo maior poder de negociação no mercado e efetuando compras individualizadas para cada país (7).

Em adição, o $45^{\circ}$ Conselho Diretor da OPAS (8) adotou, em 2004, uma resolução direcionada a promover o acesso aos medicamentos e insumos para saúde pública na Região das Américas. Como elemento chave, os Estados-Membros respaldaram a proposta de fortalecer os mecanismos regionais de aquisição, incluindo o Fundo Estratégico. O Conselho Diretor assinalou que o desenvolvimento desse fundo poderia incrementar a capacidade dos países em matéria de aquisições, programação e planejamento, permitindo economias de escala mediante a unificação das demandas e fomentando a continuidade do fornecimento dos insumos estratégicos por um sistema de aquisições cíclicas.

Em 2005, elaboraram-se os princípios operacionais do Fundo, com base no modelo de convênio entre os EstadosMembros da OPAS (6). Nessa direção, buscou-se agregar valor aos países participantes em três importantes direções: concentrando o apoio técnico aos países no planejamento das aquisições, distribuição e na previsão da demanda futura de produtos; garantindo o fornecimento de produtos de qualidade mediante aplicação de normas para aquisição de produtos farmacêuticos e de controle da qualidade; e facilitando a comunicação e a coordenação entre os provedores e os países participantes, a fim de melhorar a disponibilidade e a acessibilidade aos produtos.

Para a aquisição e a gestão dos insumos estratégicos, o apoio prevê a formulação e a revisão de planos de aquisição, o fortalecimento da capacidade para programar necessidades, estimativas das demandas futuras, financiamento, aquisição, fixação de preços e análise de fatores vinculados aos direitos de propriedade intelectual, aquisições utilizando os mecanismos e as normas do Fundo Estratégico, avaliação, monitoramento e apoio às cadeias de produção e distribuição e integração com outros mecanismos mundiais de aquisição.

Quanto à qualidade, o apoio abrange a determinação dos requisitos que os fornecedores (pré-qualificados) devem cumprir para assegurar a qualidade dos medicamentos, o fortalecimento da capacidade dos laboratórios nacionais de controle da qualidade, o apoio técnico para avaliar e assegurar a garantia da qualidade dos produtos adquiridos, o intercâmbio de experiências com outros países no processo de aquisição e a negociação dos preços dos medicamentos.

Os programas técnicos do Fundo Estratégico promovem ainda a disponibilidade e a acessibilidade contínua a insumos estratégicos tais como os medicamentos para a $\mathrm{HIV} / \mathrm{Aids}$, incluídos os antirretrovirais (ARV), em nome dos Estados-Membros. Certas atividades enfocam as negociações regionais dos preços dos ARV, o aumento da capacidade de gestão de produtos farmacêuticos para os programas prioritários de saúde pública (como o projeto da Rede Amazônica de Vigilância da Resistência às Drogas Antimaláricas, a RAVREDA) e o apoio à formulação e à implementação de planos de aquisição para pedir subvenções ao Fundo Global de Combate à Aids, Tuberculose e Malária (6).

Observa-se que, embora o Fundo Estratégico tenha sua missão e seus objetivos estruturados a partir da reforma organizacional da OPAS, os recursos financeiros e técnicos para apoiar diretamente as operações nos países são limitados. Assim, em 2004, foi estabelecido um grupo de trabalho do próprio Fundo, integrado às unidades técnicas e administrativas da OPAS, visando a desenvolver, estruturar e apoiar as atividades do Fundo Estratégico, que se reúne pelo menos duas vezes por ano $(9,10)$. A estrutura operacional atual do Fundo Estratégico aparece na figura 1 .

Nesse contexto, o objetivo deste artigo é apresentar o Fundo Estratégico da OPAS como um meio facilitador para a melhoria do acesso a medicamentos e descrever o seu processo de funcionamento.

\section{MATERIAIS E MÉTODO}

Estudo de caso retrospectivo do tipo descritivo e exploratório, com recorte temporal de 2004 a 2007. A estratégia de investigação esteve fundamentada em levantamento bibliográfico e documental em arquivos diversos. Dentre os arquivos, há de se destacar a disponibilização de dados e informações pelas representações da OPAS nos Estados Unidos (Washington D.C.), El Salvador, Guatemala, Honduras e Brasil, através do Centro LatinoAmericano e do Caribe de Informação em Ciências da Saúde (BIREME).

Para garantir a obtenção das informações mais recentes, foram coletados dados do sistema de compras do Escritório Central da OPAS nos Estados Unidos. Foram levantados o volume total de recursos e os tipos de medicamentos movimentados por meio do Fundo Estratégico nos anos de 2004, 2005, 2006 e nos meses de janeiro a setembro de 2007.

\section{RESULTADOS}

\section{Aquisição de produtos por meio do Fundo Estratégico}

As aquisições a pedido de um participante são coordenadas conjuntamente com os ministérios da saúde. Quando 
FIGURA 1. Estrutura operacional do Fundo Estratégico para medicamentos da Organização PanAmericana da Saúde ${ }^{a}$

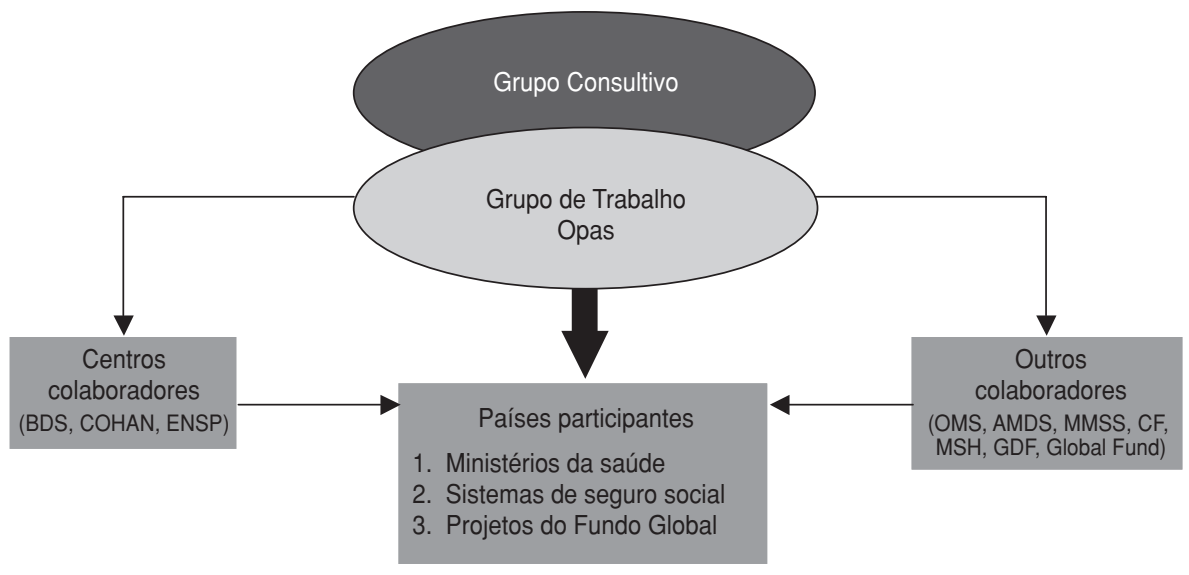

Fonte: Adaptado do Plano empresarial 2007-2010 da Organização Pan-Americana da Saúde. Escritório Central da Organização Pan-Americana da Saúde em Washington D.C., Estados Unidos, outubro de 2007.

${ }^{a}$ Centros colaboradores: BDS = Barbados Drug Service; COHAN: Hospitals Cooperative, Antioquia; ENSP: Escola Nacional de Saúde Pública. Outros colaboradores: OMS = Organização Mundial da Saúde; AMDS = AIDS Medicines and Diagnostic Service; MMSS = Malaria Medicines \& Supplies Services; CF = Clinton Foundation; MSH = Management Sciences for Health; GDF = Global Drug Facility

um país participante ou um beneficiário principal deseja realizar uma compra, o ministério da saúde ou o beneficiário principal trabalha diretamente com a representação da OPAS no país. A seleção de produtos e a determinação da demanda futura, bem como o pedido de aquisição, são feitos pela representação da OPAS, segundo o regulamento para aquisições do Fundo Estratégico.

Com base na lista de necessidades estimadas pelos Estados-Membros e programadas em conjunto com a OPAS, são estabelecidos contratos com os fornecedores pré-qualificados de insumos estratégicos. Os preços pactuados são informados previamente a todos os países envolvidos.

A entrega dos insumos estratégicos para a saúde pública é autorizada pela OPAS mediante conclusão satisfatória da análise de qualidade levada a cabo por um laboratório (centro colaborador) designado pela OPAS, de acordo com o conjunto de critérios definidos pelas Normas e Procedimentos Farmacêuticos da OMS. Os protocolos com os resultados do controle de qualidade para cada lote do respectivo insumo são enviados aos participantes do Fundo Estratégico no momento da sua distribuição. Os produtos que não corresponderem às especificações de referência da OMS ficam sob a responsabilidade do fornecedor, que deve garantir os trâmites correspondentes ao regulamento internacional. A liberação na alfândega e o pagamento de qualquer taxa alfandegária é de total responsabilidade do país que participa do Fundo Estratégico. $\mathrm{O}$ país pode realizar, dentro de um prazo pré-estabelecido, suas próprias análises da qualidade após o recebimento do material, conforme previamente pactuado com a OPAS.

\section{Gestão técnica e administrativa}

A gestão administrativa do Fundo Estratégico fica a cargo de setores administrativos da OPAS, sob a direção do gabinete do diretor administrativo na sede da Organização em Washington, nos Estados Unidos. Cada setor tem funções específicas: a Unidade de Serviços de Compras encarrega-se do processo de aquisição, facilita o envio dos produtos aos destinatários e revisa e autoriza o pagamento das faturas dos provedores; a Unidade Financeira credita os fundos na conta dos Estados-Membros, efetua o pagamento dos fornecedores, mantém as contas em dias, executa o controle financeiro, emite as faturas para as compras e fornece as informações financeiras aos interessados; a Unidade de Assuntos Jurídicos trata de questões relacionadas aos convênios com os Estados-Membros, aprova os documentos de licitações e apoia a redação de convênios contratuais e demais assuntos jurídicos.

As atividades do Fundo Estratégico especificamente o planejamento, a aqui- sição e a garantia da qualidade dos produtos - são coordenadas por três áreas técnicas da OPAS: a Área de Prevenção e Controle de Enfermidades, com suporte específico em áreas de medicamentos e insumos usados no tratamento e controle de doenças transmissíveis, como a tuberculose, a malária e leishmaniose; a Área de Saúde Familiar e Comunitária, com ênfase nos medicamentos e nos meios de diagnóstico relacionados à infecção por HIV/Aids; e a Área de Tecnologia e Prestação de Serviços de Saúde, com ênfase nos aspectos de gestão dos insumos, qualidade e uso racional de medicamentos essenciais e tecnologias de saúde.

\section{Gestão financeira}

A gestão financeira do Fundo Estratégico é regida pelas normas e regulamentos financeiros da OPAS. Os EstadosMembros e o beneficiário principal que participam do Fundo Estratégico depositam recursos em uma conta da OPAS para aquisição de produtos. Ao receber um pagamento de um Estado-Membro ou de um beneficiário principal, a OPAS se apropria dos recursos em uma conta independente para o Estado-Membro, a partir da qual são pagas as faturas aos provedores. Todo o saldo é retido nessa conta para futuras aquisições pelo Estado-Membro ou reembolsado, se assim o Estado exigir.

Todos os Estados-Membros da OPAS podem fazer parte do Fundo Estratégico, desde que aportem uma "contribuição inicial de adesão", a ser estabelecida pelo Diretor da OPAS/OMS. Essa contribuição não tem um valor mínimo. Para garantir a continuidade da operação do Fundo, é estabelecida uma contacorrente para receber as contribuições dos Estados-Membros, chamada de conta de capitalização.

Para cada pagamento efetuado à OPAS, o Estado-Membro faz uma contribuição adicional equivalente a $3 \%$ do custo dos produtos adquiridos, excluídos o frete e o seguro, isto é, $3 \%$ sobre o valor líquido dos insumos adquiridos por meio do Fundo Estratégico (taxa de capitalização). O encargo de 3\% é usado para aumentar o capital do Fundo Estratégico com a finalidade de facilitar aquisições futuras sem pagamento prévio pelos Estados-Membros. À medida que são feitas aquisições, aumenta o capital do Fundo. Além da capitalização mediante as aquisições, a OPAS pode, tam- 
bém, mobilizar outros recursos. Uma conta reserva é mantida para cada país até o patamar do valor da contribuição inicial da adesão. Uma vez atingido esse limite, todos os valores excedentes são ficam disponíveis aos seus participantes, em base de rateio, para o estabelecimento de novas ordens de compra.

\section{Análise de compras}

Os valores apresentados englobam todos os recursos executados por meio do Fundo Estratégico até setembro de 2007, independentemente da fonte de financiamento (recursos governamentais ou Fundo Global).

Os planos de aquisição incorporam preços de referência nacionais e/ou internacionais. No caso da utilização de preços internacionais de referência, esses são ajustados levando-se em consideração fretes, impostos, seguros e outras despesas de importação. Algumas fontes de preços de referência são utilizadas creditados na conta de capitalização e

para análise e comparação dos dados apresentados pelo Fundo Estratégico: preços históricos do Fundo Estratégico da OPAS; lista de preços de ARV da Fundação Clinton; e mecanismo de notificação de preços do Fundo Global.

A tabela 1 demonstra o volume de recursos movimentados nos últimos 4 anos, a quantidade e tipo de insumos estratégicos adquiridos e a quantidade de países participantes do Fundo Estratégico. É possível observar um crescimento no volume de recursos movimentados pelo Fundo Estratégico e na adesão de países participantes.

Como mostra a tabela 1, em 2004, de 38 tipos de insumos estratégicos solicitados para compras por meio do Fundo Estratégico, apenas três medicamentos destinavam-se ao tratamento do HIV/ Aids, o que representa pouco menos do que $8 \%$ do total. Por outro lado, em 2007, considerando apenas os dados até o mês de setembro, as compras de ARV já representavam 57,89\%. A figura 2 apresenta a proporção de dólares gastos com

TABELA 1. Compras efetuadas pelo Fundo Estratégico para medicamentos da Organização PanAmericana da Saúde

\begin{tabular}{|c|c|c|c|c|}
\hline & \multicolumn{4}{|c|}{ Ano } \\
\hline & 2004 & 2005 & 2006 & $2007^{a}$ \\
\hline Valor total de compras efetuadas (US\$) & 3475043 & 8328009 & 13905000 & 19646634 \\
\hline Gasto com antirretrovirais (US\$) & 11896 & 2612585 & 6935011 & 13091509 \\
\hline No. total de insumos comprados & 38 & 67 & 157 & 76 \\
\hline Tipos de antirretrovirais comprados & 3 & 33 & 62 & 44 \\
\hline No. de países participantes & 3 & 5 & 14 & 17 \\
\hline No. de países que compraram ARV & 3 & 5 & 11 & 9 \\
\hline
\end{tabular}

Fonte: Escritório Central da Organização Pan-Americana da Saúde em Washington D.C., Estados Unidos, outubro de 2007. ${ }^{a}$ De janeiro a setembro.

FIGURA 2. Evolução nas aquisições de antirretrovirais em relação à quantidade total de insumos pelo Fundo Estratégico para medicamentos da Organização Pan-Americana da Saúde

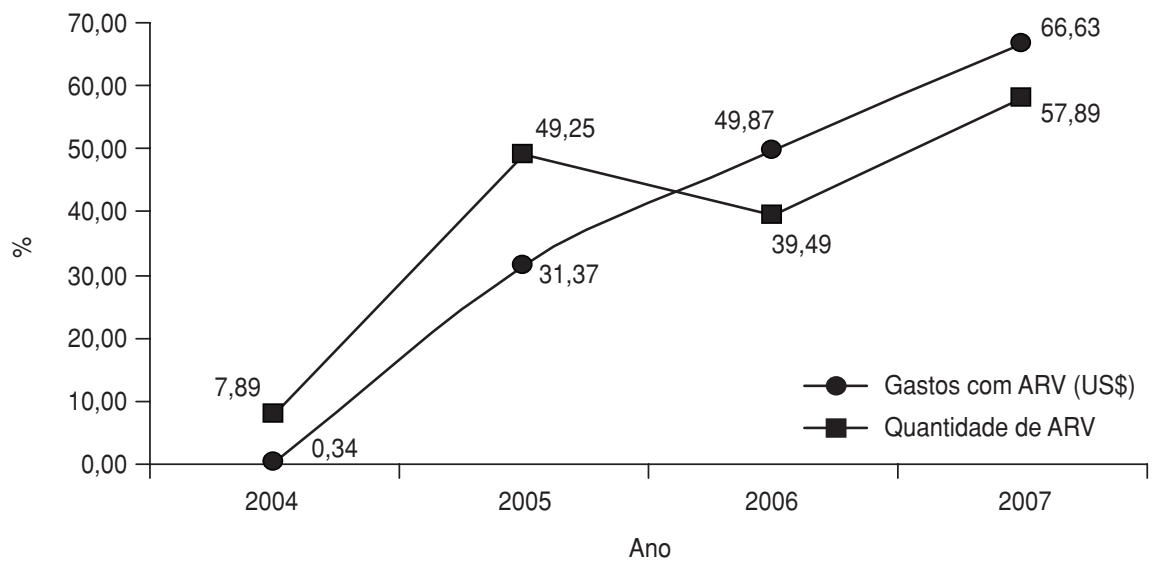

Fonte: Escritório Central da Organização Pan-Americana da Saúde, Washington D.C., Estados Unidos, 2007.
ARV em relação ao total da compra de medicamentos.

$\mathrm{O}$ percentual ascendente de gastos com ARV não se relaciona diretamente com a quantidade de medicamentos comprados. A exemplo dos dados do ano de 2006, verifica-se que foi obtida uma quantidade menor de tipos de ARV comparados aos 2 anos anteriores, porém o gasto continua sendo crescente.

A partir dos dados fornecidos pelo Escritório Central da OPAS, constatou-se que $66,63 \%$ da movimentação financeira do Fundo Estratégico, em 2007, foram destinados à compra de ARV para nove países (Brasil, Guatemala, El Salvador, Equador, Honduras, Haiti, Belize, Nicarágua e Bolívia) de um total de 17 países participantes. Ou seja, os medicamentos ARV apresentam-se com um expressiva demanda frente aos demais insumos estratégicos do Fundo. A tabela 2 mostra a distribuição dos recursos despendidos de janeiro a setembro de 2007. Além disso, o gasto com cada um dos nove países que utilizaram o Fundo Estratégico de janeiro a setembro de 2007 foi: US\$ 12353452,90 para o Brasil; US\$ 3650015,66 para a Guatemala; US\$ 1269805,49 para El Salvador; US\$ 840778,47 para o Equador; US\$ 722285,73 para Honduras; US\$ 652 143,02 para o Haiti; US\$ 135 043,74 para Belize; US\$ 13 900,00 para a Nicarágua; e US\$ 9 209,50 para a Bolívia.

Observa-se que o país que mais movimentou recursos financeiros por meio do Fundo Estratégico foi o Brasil, com $63 \%$ dos gastos para compra de insumos estratégicos, até setembro de 2007. A existência do Programa Nacional de DST/Aids do Ministério da Saúde, que possui uma grande estrutura e incentivo do Banco Mundial, pode explicar esse volume, além das facilidades do processo de compras do Fundo Estratégico por meio de Termos de Cooperação, frente às regras públicas brasileiras de aquisição de insumos estratégicos para a saúde.

Guatemala, El Salvador, Equador e Honduras foram os quatro países mais ativos nas compras pelo Fundo Estratégico em 2007, movimentando praticamente o restante dos recursos financeiros do Fundo, correspondente ao volume total de US\$ 6482 885,35, com perspectiva de ascensão nesta modalidade de compra.

\section{DISCUSSÃO}

A utilização adequada dos recursos disponíveis no Fundo Estratégico da 
TABELA 2. Distribuição dos recursos despendidos pelo Fundo Estratégico para medicamentos da Organização Pan-Americana da Saúde de janeiro a setembro de $2007^{\mathrm{a}}$

\begin{tabular}{lr}
\hline \multicolumn{1}{c}{ Item } & \multicolumn{1}{c}{ Valor (US\$) } \\
\hline Antirretrovirais & 13050705,74 \\
Inseticidas & 4240576,66 \\
Medicamentos para tuberculose & 1433930,86 \\
Outros fármacos & 540169,61 \\
Medicamentos para malária & 274131,80 \\
Insumos para diagnóstico & 66316,00
\end{tabular}

Fonte: Escritório Central da Organização Pan-Americana da Saúde em Washington D.C., Estados Unidos, outubro de 2007.

a Valor total: US\$19 646634,51

OPAS não significa apenas a compra de medicamentos a um menor preço possível. Para alcançar uma maior eficiência na utilização desses recursos, a seleção dos medicamentos é fundamental. Ressalta-se que a gestão do processo de compra de medicamentos via Fundo Estratégico (9) vem sendo facilitada pelo reconhecimento internacional da OPAS como organismo internacional que apoia a assistência farmacêutica (11) e que oferece suporte para o fornecimento de medicamentos com qualidade e preços justos, considerando o ciclo da assistência farmacêutica: seleção, programação, aquisição, armazenamento, distribuição e uso racional.

$\mathrm{O}$ arcabouço desse ciclo contém um núcleo formado por um sistema de apoio administrativo: organização, financiamento, sustentabilidade, gestão da informação e administração de pessoal. Esse núcleo deve manter a integridade da gestão de medicamentos, que deve ser fundamentada em uma política e em uma estrutura legal que estabeleçam e mantenham o compromisso de um fornecimento de medicamentos públicos com qualidade.

Os componentes estratégicos que permitem uma gestão adequada de fornecimento de medicamentos são o planejamento e o controle. Um planejamento eficaz requer uma reflexão atenta sobre os objetivos básicos e uma avaliação sistemática da situação existente. O controle contínuo é uma avaliação periódica para medir o progresso alcançado, ajustar o plano de implantação e avaliar a repercussão das melhorias realizadas na gestão de medicamentos. Ademais, a definição de indicadores, objetivos e metas específicas para os programas proporcionam medidas concretas que permitem comparação com os resultados reais alcançados.

Assim, as experiências reunidas de muitos países e de numerosos programas de saúde demonstram que é possível conseguir melhoras consideráveis e sustentáveis no fornecimento de medicamentos e insumos estratégicos para a saúde. Porém, o êxito não está assegurado. Um objetivo claro, um plano sólido, colocado em prática de forma eficaz, com um controle sistemático dos resultados, são ingredientes essenciais ao desenvolvimento do setor farmacêutico, em especial ao processo de aquisição de insumos estratégicos essenciais.

\section{Conclusão}

Como já foi mencionado, os desafios que se apresentam para nossos sistemas e serviços de saúde no sentido de asse-

\section{REFERÊNCIAS}

1. Bermudez J. Acesso a insumos em saúde: desafios para o Terceiro Mundo. Cad Saude Publica. 2007;23(4):744-5.

2. Jadue L, Delgado I, Sandoval H, Cabezas L, Vega J. Análisis del nuevo Módulo de Salud de la Encuesta CASEN 2000. Rev Med Chil. 2004;132(6):750-60.

3. World Health Organization. Constitution of the World Health Organization. Am J Pub Health. 1946;1315-6. Disponível em: www. pubmedcentral.nih.gov/picrender.fcgi?artid $=1625885 \&$ blobtype $=$ pdf. Acessado em outubro de 2009.

4. Organización Mundial de la Salud. Acceso equitativo a los medicamentos esenciales: un marco para la acción colectiva. Genebra: OMS; 2004. (Perspectivas políticas de la OMS sobre medicamentos). Disponível em: apps. who.int/medicinedocs/en/d/Js4964s/1.html. Acessado em outubro de 2009. gurar o acesso aos insumos essenciais se confrontam com um padrão de iniquidade que nos transforma em uma região de desequilíbrio, de extrema concentração de renda e de injustiças evidentes. Nesse contexto, infere-se que o Fundo Estratégico, na tentativa de minimizar a iniquidade no acesso aos serviços de saúde, tem alcançado:

- o fortalecimento da capacidade dos Países-Membros para programação, planejamento e aquisição, facilitando o avanço das economias de escala ao consolidar a demanda do produto, promovendo a continuidade na provisão através do desenvolvimento de um sistema aquisitivo integrado;

- a coerência com objetivos, aspirações e compromissos das políticas farmacêuticas nacionais que procuram assegurar o acesso equitativo, a qualidade e o uso racional dos medicamentos;

- a eficiência nas compras farmacêuticas, investindo numa administração eficaz e transparente por meio da seleção de medicamentos essenciais, processo de planejamento e quantificação adequados, financiamento, competição e identificação de fornecedores com garantia da qualidade.

Dessa forma, o Fundo tem contribuído para incrementar a capacidade nacional em relação à aquisição e ao controle da qualidade dos produtos e à aplicação das normas de garantia da qualidade e gestão administrativa das aquisições, demonstrando ser uma estratégia vantajosa. Por fim, é importante salientar que são necessários estudos para avaliar o impacto da participação do Fundo Estratégico na melhoria do acesso aos medicamentos essenciais e insumos estratégicos nos Estados-Membros.

Agradecimentos. À Organização PanAmericana da Saúde no Brasil.
5. Pan American Health Organization. Regional revolving fund for strategic public health supplies. 42nd Directing Council of the Pan American Health Organization. Washington DC: PAHO/OMS; 2000. Disponível em: www.paho.org/English/gov/cd/cd42_30-e. pdf. Acessado em outubro de 2009.

6. Pan American Health Organization. Operating principles for the regional revolving fund for strategic public health supplies: a PAHO 
mechanism for procurement of strategic public health supplies. Genebra: PAHO; 2006. Disponível em: www.paho.org/English/AD/ THS/EV/Operating_Principles_English.pdf. Acessado em outubro de 2009.

7. World Health Organization. WHO medicines strategy: framework for action in essential drugs and medicines policy. Genebra: WHO; 2000.

8. Pan American Health Organization. 45th Directing Council. 56th Session of the Regional Committee. Washington D.C.: PAHO; 2004. Disponível em: www.paho.org/english/gov/
cd/CD45-10-e.pdf. Acessado em outubro de 2009.

9. Organización Mundial de la Salud. Informe sobre la cooperación tecnica que desarrolla la OPS a través de Fondo Estratégico en apoyo al suministro de medicamentos al Ministerio de Salud de El Salvador. Genebra: OMS; 2005. Disponível em: http:/ /amro.who.int/spanish/ ad/ths/ev/Mini-boletin_007.pdf. Acessado em outubro de 2009.

10. Organización Panamericana de la Salud. Salud en las Américas. Volumen II - países. Washington: OPAS; 2007. Disponível em: www.paho.org/hia/vol2paises.html. Acessado em outubro de 2009.

11. Brasil, Ministério da Saúde. Portaria GM no. 3916. Política Nacional de Medicamentos. Brasília: Centro de Documentação; 1998. Disponível em: www.anvisa.gov.br/legis/ consolidada/portaria_3916_98.pdf. Acessado em outubro de 2009.

Manuscrito recebido em 27 de junho. Aceito em versão revisada em 12 de maio de 2009

ABSTRACT Objective. To describe the medicine procurement activities of the PAHO Strategic Fund.

The PAHO Strategic Fund: a mechanism to facilitate access to medicines

Key words
Method. This is a retrospective study covering the period from 2004 to 2007, based on a bibliographic and document survey of Pan American Health Organization (PAHO) archives in the United States, El Salvador, Guatemala, Honduras, and Brazil. The volume of resources and the types of drugs procured by the fund were determined for the years 2004, 2005, and 2006 and for the period of January to September 2007.

Results. The survey revealed a well-structured fund handling increasing resources, from US\$ 3475043.00 in 2004 to US\$ 19646634.00 in 2007 (January to September). The participation of antiretroviral drugs in this expenditure has grown from less than $8 \%$ of the total in 2004 to $57.89 \%$ in 2007 . Still, in $2007,66.63 \%$ of the financial resources managed by the Strategic Fund were allocated to the purchase of antiretroviral drugs for nine countries (Brazil, Guatemala, El Salvador, Ecuador, Honduras, Haiti, Belize, Nicaragua, and Bolivia) out of a total of 17 participating countries. Brazil was the country using the most resources through the Strategic Fund, accounting for $63 \%$ of the expenditure for the purchase of strategic supplies between January and September 2007.

Conclusion. The proposal to unify the purchase of medications for all participating countries to improve pricing and purchasing management will result in large-scale savings. The Strategic Fund can contribute to increasing access to medicines and improving the management of the public health care system in Latin America.

Drugs, essential; technical cooperation; equity in access; Latin America. 